\title{
Humeans Aren't Out of Their Minds
}

\author{
Brian Weatherson
}

Humeanism is "the thesis that the whole truth about a world like ours supervenes on the spatiotemporal distribution of local qualities." (Lewis, 1994, 473) Since the whole truth about our world contains truths about causation, causation must be located in the mosaic of local qualities that the Humean says constitute the whole truth about the world. The most natural ways to do this involve causation being in some sense extrinsic. To take the simplest possible Humean analysis, we might say that $c$ causes $e$ iff throughout the mosaic events of the same type as $c$ are usually followed by events of type $e$. For short, the causal relation is the constant conjunction relation. Whether this obtains is determined by the mosaic, so this is a Humean theory, but it isn't determined just by $c$ and $e$ themselves, so whether $c$ causes $e$ is extrinsic to the pair. Now this is obviously a bad theory of causation, but the fact that causation is extrinsic is retained even by good Humean theories of causation. John Hawthorne (2004) objects to this feature of Humeanism. I'm going to argue that his arguments don't work, but first we need to clear up three preliminaries about causation and intrinsicness.

First, my wording so far has been cagey because I haven't wanted to say that Humeans typically take causation to be an extrinsic relation. That's because the greatest Humean of them all, David Lewis, denies that causation is a relation at all, and hence that it is an extrinsic relation (Lewis, 2004b). We can go some way to avoiding this complication by talking, as Hawthorne does, about properties of regions, and asking the property of containing a duplicate of $c$ that causes a duplicate of $e$ is intrinsic or extrinsic. ${ }^{1}$ Humeans typically take causation to be extrinsic in this sense.

Second, nothing in Humeanism requires that causation is extrinsic in that sense. If one analysed causation as that intrinsic relation that actually most tightly correlates with the constant conjunction relation, then one would have guaranteed that causation was an intrinsic relation. Moreover, one would have a perfectly Humean theory of causation. (A perfectly awful theory, to be sure, but still a Humean one.) Peter Menzies (1996, 1999) develops a more sophisticated version of such a theory, and though Menzies describes his view as anti-Humean, one can locate the relation we've defined here in the Humean mosaic, so such an approach might be consistent with Humeanism in the intended sense.

Third, there is good reason, independent of Humeanism, to accept that causation is extrinsic. As Ned Hall (2004) argues, it is very hard to square the intrinsicness of causation with the possibility of causation by omission. Given the choice between

\footnotetext{
$\dagger$ Penultimate draft only. Please cite published version if possible. Final version published in Noûs 41 (2007): 529-535.

${ }^{1}$ This move requires the not wholly uncontroversial assumption that regions are the kinds of things that can have properties. But I'll happily make that assumption here. Note that the formulation here allows that the property denoted might be intrinsic for some $c$ and $e$ and extrinsic for others. I'll say causation is extrinsic if the property denoted is extrinsic for some choice of $c$ and $e$, even if it is intrinsic for others, as it might be if, for example, no region could possess the property because $c$ is a part of $e$.
} 
these two, I'm going to accept causation by omission without much hesitation. There is one powerful objection to the possibility of causation by omission, namely that if there is any causation by omission then there is a lot more than is intuitively plausible. But since Sarah McGrath (2005) has a good response to that objection, I feel happy accepting there is causation by omission. So I accept that causation is extrinsic, for reasons totally independent of Humeanism. Since Hawthorne appeals to no feature of Humeanism beyond the Humean's acceptance of the extrinsicness of causation, we can take his argument to be an argument against the causal extrinsicalist, i.e. the theorist who accepts causation is extrinsic in the above sense. To see that the argument doesn't go through, we need to consider what exactly the causal extrinsicalist is committed to. I'll explore this by looking at some other examples of properties of regions.

Some regions contain uncles and some do not. This seems to be an extrinsic property of regions. My house does not contain any uncles right now, but there are duplicates of it, in worlds where my brothers have children, where it does contain an uncle, namely my counterpart. Consider the smallest region containing the earth from the stratosphere in from the earth's formation to its destruction. Call this region $e$. Any duplicate of $e$ also contains uncles, including several uncles of mine. You can't duplicate the earth without producing a duplicate of me who is, in the duplicate world, the nephew of the duplicates of my uncles. So it is intrinsic to $e$ that it contain an uncle, even though this is an extrinsic property of regions. (There is much more discussion of extrinsic properties that are possessed intrinsically in Humberstone (1996).)

This possibility, that a region might intrinsically possess an extrinsic property, poses a problem for Hawthorne's argument. Here is his presentation of it.

(1) An intrinsic duplicate of any region wholly containing me will contain a being with my conscious life.

(2) There are causal requirements on my conscious life.

Therefore, Humeanism is false. (Hawthorne, 2004, 351-352)

The problem is that this argument isn't valid. What follows from (1) and (2) is that any region containing Hawthorne must possess some causal properties intrinsically. (As Hawthorne argues on page 356.) And what Humeanism entails is that causal properties are extrinsic properties of regions. But there is no incompatibility here, for it is possible that extrinsic properties are possessed intrinsically, as we saw in the discussion of uncles.

Hawthorne's argument would go through if Humeans, and causal extrinsicalists more generally, were committed to the stronger claim that regions never possess causal properties intrinsically. But it doesn't seem that Humeans should be committed to this claim. Consider again $e$ and all its duplicates. Any such duplicate will contain a duplication of the event of Booth's shooting Lincoln, and Lincoln dying. ${ }^{2}$

\footnotetext{
${ }^{2}$ There is a potential complication here in that arguably in some such worlds, e.g. worlds where there is another planet on the opposite side of the sun to duplicate-earth where people are immediately
} 
Will it also be the case that duplicate-Booth's shooting in this world causes duplicateLincoln's dying? If so, and this seems true, then it is intrinsic to $e$ that it contains an event of a shooting causing a dying, even though the property of containing a shooting causing a dying is extrinsic.

It would be a bad mistake to offer the following epistemological argument that in all duplicates of $e$, duplicate-Booth's shooting causes duplicate-Lincoln's dying.

1. If there was a duplicate of $e$ where duplicate-Booth's shooting does not cause duplicate-Lincoln's dying, then we would not know whether Booth's shooting causes Lincoln's dying without investigating what happens outside $e$.

2. We can know that Booth's shooting caused Lincoln's dying without investigating outside $e$.

3. So, there is no duplicate of $e$ where duplicate-Booth's shooting does not cause duplicate-Lincoln's dying.

The problem with this argument is that even there are worlds containing such duplicates, we might know a priori that we do not live in such a world, just as we know a priori that we do not live in a world where certain kinds of sceptical scenarios unfold (Hawthorne, 2002; Weatherson, 2005).

A better argument against the existence of such a world is that if it is possible, it should be conceivable. But it is basically impossible to conceive such a world. Even if throughout the universe shootings like Booth's are usually followed by something other than dying, say that shooting in most parts of the universe causes diseases to be cured, the large-scale regularity within $e$ (or its duplicate) of shootings being followed by dying suffices to ground the claim that shootings cause dyings in a good Humean theory. The crucial assumption here is that local regularities count for more than global regularities. If the local regularities deviate too far from the global regularities, then Humeans can and should say that different nomic claims (and hence causal claims) are true in this part of the world to the rest of the universe. If they say this, they can say that regions can have causal features (such as containing a shooting causing a dying) intrinsically even though causal features are extrinsic properties.

To illustrate the kind of Humean theory that would have such a consequence, consider the following variant on the constant conjunction theory of causation. The theory I'm imagining says that $c$ causes $e$ iff whenever an event of the same type as $c$ occurs within a 50 mile radius of where $c$ occurred, it was followed by an event of type e. Call this the 50 mile constant conjunction theory of causation. ${ }^{3}$ On the 50 mile constant conjunction theory of causation, it won't be intrinsic to Ford's Theatre that it contained a causal event involving Booth shooting Lincoln, but it will be intrinsic

'resurrected' when the 'die' on duplicate-earth. In such a world you might say that duplicate-Lincoln doesn't really die on duplicate-Earth, but merely has the duplicate-earth part of his life ended. We'll understand 'dying' in such a way that this counts as dying.

${ }^{3}$ I assume here that events can be properly said to have locations. Spelling out this assumption in detail will require some serious metaphysics, particularly when it comes to omissions. Where exactly does my omission to stop the Iraq War take place? Here at my kitchen table where I am? In Iraq, where the war is? In Washington, if that's where I'd be were I doing something to stop the war? These questions are hard, though not so hard that we should give up on the very natural idea that events have locations. 
to any sphere of radius 50 miles or more centred on the theatre that it contains such a causal event. So on this theory causal properties can be intrinsic to a region, though they are still extrinsic properties of such a region.

That's a very implausible Humean theory, but when we look at the details of David Lewis's Humean picture, we can see the outlines of a more plausible theory with the same consequences. Lewis of course doesn't offer a simple regularity theory of causation. Rather, he first argues that laws are the extremely simple, extremely informative true propositions (Lewis, 1973, 73). That is, he offers a sophisticated regularity theory of laws. Then he analyses counterfactual dependence in terms of lawhood (Lewis, 1973, 1979). Finally he analyses causation in terms of counterfactual dependence (Lewis, 2004a). The philosophical theory meets the Humean mosaic most closely on the issue of what a law is. If we can offer a theory of laws that allows extra sensitivity to local facts, while remaining Humean, we can plug this back into Lewis's theories concerning counterfactual dependence and hence causation without upsetting its Humean credentials.

Now there is a good reason to think that a Humean theory of laws should be locally sensitive. (I'm indebted here to long ago conversations with James Chase.) Humeans typically believe in fairly unrestricted principles of recombination. And they believe that laws are not necessarily true. So there could be worlds with very different laws. So there is a world which 'patches' together part of the world with laws $L_{1}$ with a world with laws $L_{2}$. If the parts are large and isolated enough, it would be foolish to say that within those parts nothing is law-governed, or that within those regions there is no counterfactual dependence, or no causation. Much better to say that regularities obtaining within such a region are sufficiently simple and informative to count as laws. In our patchwork world, the laws might simply say In $r_{1}, L_{1}$ and in $r_{2}, L_{2}$. Provided the terms denoting the regions are not too gruesome, these will plausibly be Humean, even Lewisian, laws.

Let's bring all this back to Hawthorne's example. Hawthorne argues that certain causal facts are intrinsic to the region containing his body. The challenge for the Humean is to say how this could be so when Hawthorne could be embedded in a world where very different regularities obtain. The simple answer is to say that in such worlds, laws like $I n r, L$, where $r$ picks out the region Hawthorne's body occupies, and $L$ picks out a real-world law, will be true, simple and informative. It is informative because any duplicate of Hawthorne's body is a very complicated entity, containing billions of billions of particles interacting in systematic ways, ways that are nicely summarised by real-world laws. Simplicity is a little harder to make out, but note that there is a reasonably sharp boundary between Hawthorne's body and the rest of the world (Lewis 1993), so there should be a natural enough way to pick it out. In other words, even if we embed a Hawthorne duplicate in a world with very different regularities, Humeans will still have good reason to say that the laws, and hence the facts about counterfactual dependence and causation, inside that duplicate are not changed. So not only is it logically possible that Hawthorne's premises are true and his conclusion false, we can motivate a Humean position that endorses the truth of Hawthorne's premises and the falsity of the conclusion. 
Since Hawthorne's argument is invalid then, we can accept the premises without giving up Humeanism. But I think it is worthwhile to note that his (1) also can be questioned. Hawthorne notes that it is rejected by those such as Dretske and Lewis who say that phenomenal character is determined in part by kind membership. (See Lycan (2001) for a longer defence of this kind of rejection of (1).) Hawthorne thinks that the intuitive plausibility of (1) constitutes a serious objection to those views. But by reflecting a little on the phenomenology of what I'll call totality qualia, we can undermine the intuitive case for (1).

Tweedledee is facing a perfectly symmetrical scene. His visual field is symmetric, with two gentle mountains rising to his left and his right and a symmetric plain in between them. All he can hear are two birds singing in perfect harmony, one behind his left ear and one behind his right ear. The smells of the field seem to envelope him rather than coming from any particular direction. There is a cool breeze blowing directly on his face. It's a rather pleasant scene, and the overwhelming feeling is one of symmetry.

Tweedledum is very much like Tweedledee. Indeed, Tweedledum contains a duplicate of Tweedledee as a proper part. But Tweedledum also has some sensors in his skin, and brain cells in what corresponds to a suspiciously empty part of Tweedledee's brain, that allow him to detect, and feel, where the magnetic fields are in the vicinity. And sadly, though Tweedledum is facing a duplicate of the scene facing Tweedledee, there is a major disturbance in the magnetic field just to Tweedledum's left. This produces a jarring sensation in Tweedledum's left side. As a consequence, Tweedledum does not share Tweedledee's feeling of symmetry.

Whether a picture is symmetric is a property of its internal features, but it is also a feature that can be destroyed without changing the internal features by just adding more material to one side. It is a totality property of pictures, a property the picture has because it stops just where it does. ${ }^{4}$ Similarly, totality qualia are qualia that we have in part because we don't have any more feelings than we actually do. Feelings of symmetry are totality qualia in this sense, as are many of the feelings of calm and peacefulness associated with Tweedledee's state. It is not intuitive that totality qualia should be intrinsic to a region. Indeed, it seems intuitive that a duplicate of me that was extended to produce more sensory features would lack these feelings. Hence a duplicate of me would not share my conscious life in all respects, so Hawthorne's premise (1) is also false. To be sure, these totality qualia are a somewhat speculative suggestion, but the Humean does not need them since Hawthorne's anti-Humean argument is invalid.

\section{References}

Hall, Ned, (2004). "Causation and the Price of Transitivity." In John Collins, Ned Hall, and L. A. Paul (eds.), Causation and Counterfactuals, 181-203. Cambridge: MIT Press. (1)

\footnotetext{
${ }^{4}$ Ted Sider $(2001,2003)$ stresses the importance to a theory of intrinsicness of properties that are instantiated in virtue of the object not bearing relations to other objects. My example here is closely modeled on examples from his papers.
} 
Hawthorne, John, (2002). "Deeply Contingent A Priori Knowledge." Philosophy and Phenomenological Research 65: 247-269, doi:10.1111/j.19331592.2002.tb00201.x. (3)

-, (2004). "Humeans Are Out of Their Minds." Noûs 38: 351-358, doi:10.1111/j.1468-0068.2004.00473.x. (1, 2)

Humberstone, I. L., (1996). "Intrinsic/Extrinsic." Synthese 108: 205-267, doi:10.1007/bf00413498. (2)

Lewis, David, (1973). Counterfactuals. Oxford: Blackwell Publishers. (4)

—, (1979). "Counterfactual Dependence and Time's Arrow." Nồs 13: 455-476, doi:10.2307/2215339. Reprinted in Philosophical Papers, Volume II, pp. 32-52. (4)

-, (1994). "Humean Supervenience Debugged." Mind 103: 473-490, doi:10.1093/mind/103.412.473. Reprinted in Papers in Metaphysics and Epistemology, pp. 224-247. (1)

—, (2004). "Causation as Influence." In John Collins, Ned Hall, and L. A. Paul (eds.), Causation and Counterfactuals, 75-106. Cambridge: MIT Press. (4)

-, (2004). "Void and Object." In John Collins, Ned Hall, and L. A. Paul (eds.), Causation and Counterfactuals, 277-290. Cambridge: MIT Press. (1)

Lycan, William, (2001). “The Case for Phenomenal Externalism.” Philosophical Perspectives 15: 17-35, doi:10.1111/0029-4624.35.s15.2. (5)

McGrath, Sarah, (2005). "Causation by Omission: A Dilemma." Philosophical Studies 123: 125-148, doi:10.1007/s11098-004-5216-z. (2)

Menzies, Peter, (1996). "Probabilistic Causation and the Pre-emption Problem." Mind 105: 85-117, doi:10.1093/mind/105.417.85. (1)

-, (1999). "Intrinsic versus Extrinsic Conceptions of Causation." In Howard Sankey (ed.), Causation and Laws of Nature, 313-329. Dordrecht: Kluwer. (1)

Sider, Theodore, (2001). "Maximality and Intrinsic Properties." Philosophy and Phenomenological Research 63: 357-364, doi:10.1111/j.1933-1592.2001.tb00109.x. (5)

-, (2003). "Maximality and Microphysical Supervenience." Philosophy and Phenomenological Research 66: 139-149, doi:10.1111/j.1933-1592.2003.tb00247.x. (5)

Weatherson, Brian, (2005). "Scepticism, Rationalism and Externalism." Oxford Studies in Epistemology 1: 311-331. (3) 